\title{
Blood Hormones and Venous Thromboembolic Events: Lack of Association or Lack of Standardization?
}

\author{
Daniele Pastori $^{1} \quad$ Francesco Violi $^{1}$ \\ ${ }^{1}$ Department of Internal Medicine and Medical Specialties, Sapienza \\ University of Rome, Rome, Italy \\ Thromb Haemost 2018;118:1847-1849.
}

Address for correspondence Francesco Violi, MD, I Clinica Medica, Department of Internal Medicine and Medical Specialties, Sapienza University of Rome, Viale del Policlinico 155, 00161 Rome, Italy (e-mail: francesco.violi@uniroma1.it; daniele.pastori@uniroma1.it).
Hormone replacement therapy (HRT) is widely used to control menopausal-related vasomotor symptoms and genitourinary syndrome, and to prevent the onset of osteoporosis and bone fractures. The benefits of HRT are most likely when it is started early in women aged $<60$ years or who are within 10 years of menopause onset. ${ }^{1}$

However, an increased risk of venous thromboembolism (VTE) has been described in patients receiving HRT, and a rapidly growing body of evidence on this argument is available. $^{2,3}$

This risk seems to be attributable to an effect of sex hormones on coagulation and fibrinolysis systems, by increasing blood coagulation factors and reducing inhibitors such as anti-thrombin and plasminogen activator inhibitor-1 (-Fig. 1). .,5 $^{4}$

Recent evidence suggested that oral versus non-oral HRT (i.e. transdermal) is associated with an increased risk of VTE with an odds ratio (OR) of 1.66 (95\% confidence interval [CI], 1.39-1.98). ${ }^{2}$ This increased risk of VTE was observed both in users of oral oestrogen only (OR, 1.43, 95\% CI, 1.34-1.53) and of combined oral oestrogen-progestin HRT (OR, 2.35, 95\% CI, 1.9-2.9). ${ }^{2}$ Interestingly, non-oral HRT was not associated with VTE, regardless of the type of hormone considered. ${ }^{2}$ Regarding the type of HRT, it is known that the addition of progestin to oestrogen (opposed oestrogen) reduces the risk of ovarian cancer, ${ }^{6}$ and is currently the gold standard HRT for women with a uterus. ${ }^{7}$ Results from the Women's Health Initiative oestrogen plus progestin clinical trial showed that oestrogen plus progestin oral administration was associated with a doubled risk of VTE. ${ }^{8}$ In particular, the incidence of venous thrombosis was 3.5 and 1.7 per 1,000 person-years in women taking oestrogen plus progestin or placebo, respectively (hazard ratio [HR], 2.06, 95\% CI, 1.57-2.70). This risk increased by aging (HR, 7.46, 95\% CI, 4.32-14.38 for age 7079 years), and with body weight (HR, 5.61, 95\% CI, 3.12-10.11 for obesity). ${ }^{8}$ Of note, the presence of factor V Leiden was the only factor that increased the hormone-associated risk of thrombosis by 6.69 -fold (95\% CI, 3.09-14.49), while other mutations (pro-thrombin 20210A, methylenetetrahydrofolate reductase C677T, factor XIII Val34Leu, PAI-1 4G/5G and factor V HR2) did not confer such risk. ${ }^{8}$

In this issue of the journal, ${ }^{9}$ Roetker et al performed an analysis from the Atherosclerosis Risk in Communities (ARIC) Study to investigate whether circulating blood levels of endogenous hormones, such as dehydroepiandrosterone sulphate (DHEAS), testosterone and sex hormone-binding globulin (SHBG), were associated with the incidence of VTE in men and post-menopausal women receiving HRT or no HRT. The study included 3,051 women not on HRT, 1,414 women on oral HRT and 3,925 men. ${ }^{9}$ During a follow-up of 17.6 years, 184 (6.0\%), 62 (4.4\%) and 206 (5.2\%) VTE events were recorded in the three groups, respectively. ${ }^{9}$

The authors found no association between endogenous levels of DHEAS, testosterone and SHBG with VTE incidence in men and women not using HRT, suggesting a lack of interference between blood levels of endogenous hormones and VTE risk. In addition, in women on HRT, a possible association was found for very low levels of DHEAS and a trend for very high concentrations of SHBG. However, there are some issues to be considered when analysing the results of this report. Thus, it should be taken into consideration that the population included in the ARIC Study seems to be at 'low risk' for VTE, as patients with previous VTE and cancer were excluded. Another issue is that plasma hormones were measured only at baseline with a direct immunoassay, which is less specific and sensitive than liquid chromatographymass spectrometry assay and has high coefficients of variations, as correctly acknowledged by the authors. Finally, no information regarding the type of HRT and time of exposure received

September 19, 2018

accepted after revision

September 25, 2018 (c) 2018 Georg Thieme Verlag KG Stuttgart · New York
DOI https://doi.org/ 10.1055/s-0038-1675176. ISSN 0340-6245. 


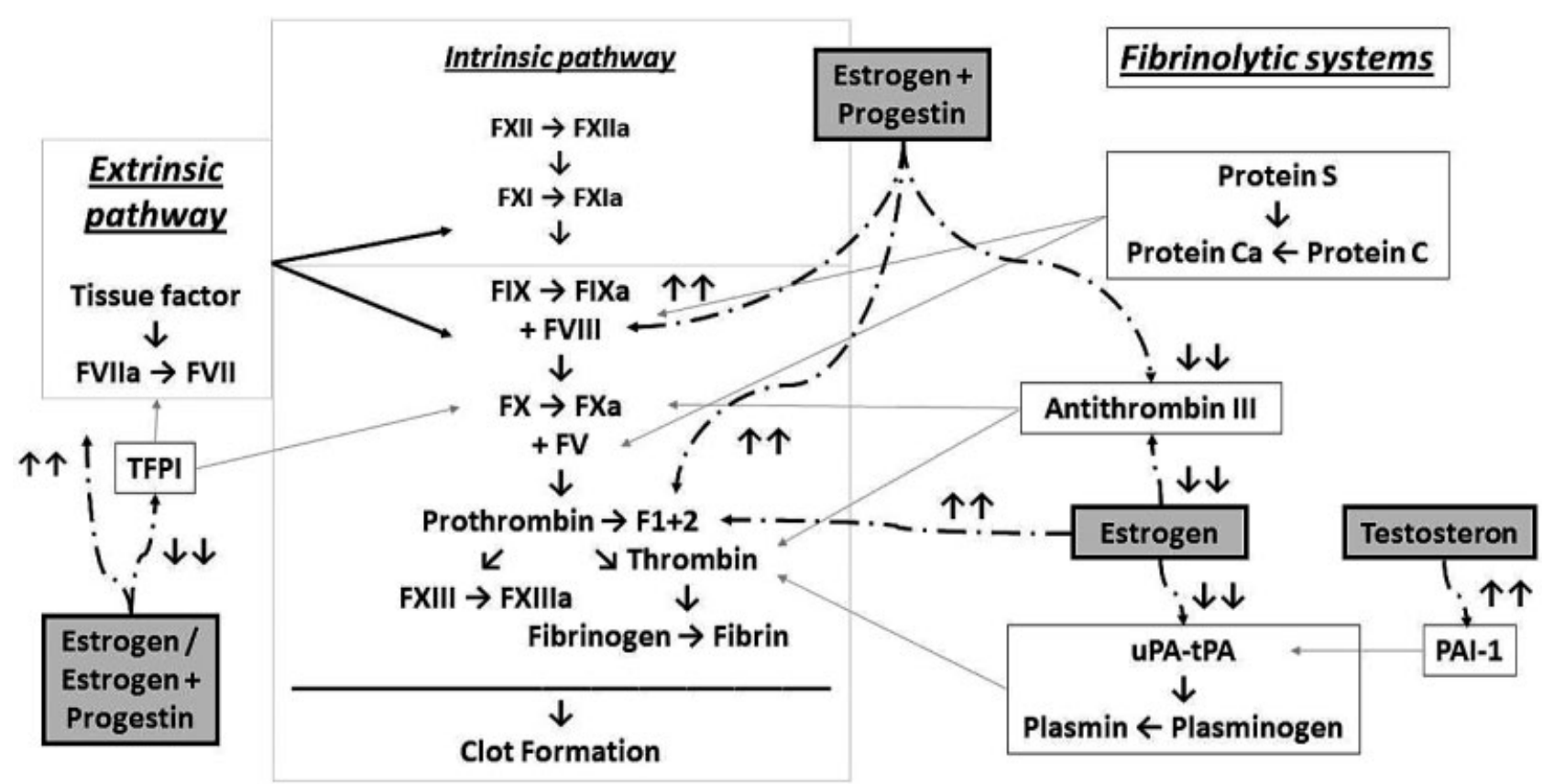

Fig. 1 Effect of sex hormones on coagulation and fibrinolysis systems. F, factor; F1 + 2, pro-thrombin fragment $1+2$; PAl-1, plasminogen activator inhibitor-1; TFPI, tissue factor pathway inhibitor; tPA, tissue plasminogen activator; uPA, urokinase.

during the follow-up period is available, which is crucial to evaluate the impact of HRT on blood hormones levels and on VTE incidence.

Another interesting issue concerns how many VTE events were potentially related to new-onset cancer in the ARIC Study. This is a critical issue as HRT has been shown to increase the risk of breast cancer. In a nationwide breast cancer screening program, the Breast Cancer Detection Demonstration Project, which included 46,355 post-menopausal women, 2,082 cases of breast cancer were identified during follow-up. Relative risk of breast cancer was 1.2 (95\% CI, 1.0-1.4) with oestrogen-only HRT and 1.4 (95\% CI, 1.1$1.8)$ with oestrogen-progestin, and this risk increased by 0.08 (95\% CI, 0.02-0.16) for each year of oestrogen-progestin use. The authors concluded that the oestrogen-progestin regimen increased breast cancer risk beyond that associated with oestrogen alone. ${ }^{10}$

\section{How to Identify Subjects at Risk for VTE during HRT?}

In addition to 'HRT-related' factors, such as the way of administration (i.e. oral vs. non-oral), which has emerged as an important determinant of VTE risk, and the type of HRT (i.e. oestrogen alone vs. oestrogen-progestin), there are also 'patients-related' variables influencing the risk of VTE. Similarly to unprovoked VTE, some factors may increase the risk of future VTE, such as aging, overweight/obesity, ${ }^{11}$ smoking, the presence of previous VTE events, recent surgery, prolonged immobilization or other co-morbidities such as active cancer, ${ }^{12}$ acquired/inherited thrombophilia conditions (i.e. autoimmune diseases, ${ }^{13}$ factor $\mathrm{V}$ Leiden ${ }^{14}$ ) and, more recently, non-O blood groups. ${ }^{11}$
However, so far, there are no risk assessment models to predict the risk of VTE in subjects under HRT. Recently, some biomarkers have been proposed to identify patients at high risk of VTE during HRT, including lower coagulative protein C and free protein $\mathrm{S}$, and also higher D-dimer, pro-thrombin fragment 1.2 and plasmin-anti-plasmin complex were found to be associated with risk of future thrombosis. ${ }^{3}$ The strongest association was found for D-dimer (OR, 6.0, 95\% CI, 3.69.8 ), and women with three or more abnormal biomarkers had 15.5-fold increased odds of VTE. ${ }^{3}$

In conclusion, the work by Roetker et $\mathrm{al}^{9}$ prospectively shows that endogenous hormone levels are not associated with VTE. However, for all the above-mentioned issues, data on this topic cannot be considered conclusive. Further studies are needed to investigate the relationship between oral/ non-oral HRT, oestrogen/oestrogen-progestin HRT, blood hormones and VTE incidence.

Conflict of Interest

None.

\section{References}

1 The NAMS 2017 Hormone Therapy Position Statement Advisory Panel. The 2017 hormone therapy position statement of The North American Menopause Society. Menopause 2017;24(07): 728-753

2 Rovinski D, Ramos RB, Fighera TM, Casanova GK, Spritzer PM. Risk of venous thromboembolism events in postmenopausal women using oral versus non-oral hormone therapy: a systematic review and meta-analysis. Thromb Res 2018;168:83-95

3 Cushman M, Larson JC, Rosendaal FR, et al. Biomarkers, menopausal hormone therapy and risk of venous thrombosis: the Women's Health Initiative. Res Pract Thromb Haemost 2018;2 (02):310-319 
4 Brodin E, Vikan T, Hansen JB, Svartberg J. Testosterone, hemostasis, and cardiovascular diseases in men. Semin Thromb Hemost 2011;37(01):87-94

5 Farris M, Bastianelli C, Rosato E, Brosens I, Benagiano G. Pharmacodynamics of combined estrogen-progestin oral contraceptives: 2. Effects on hemostasis. Expert Rev Clin Pharmacol 2017;10(10): $1129-1144$

6 Lacey JV Jr, Mink PJ, Lubin JH, et al. Menopausal hormone replacement therapy and risk of ovarian cancer. JAMA 2002;288 (03):334-341

7 Willett WC, Colditz G, Stampfer M. Postmenopausal estrogensopposed, unopposed, or none of the above. JAMA 2000;283(04): 534-535

8 Cushman M, Kuller LH, Prentice R, et al; Women's Health Initiative Investigators. Estrogen plus progestin and risk of venous thrombosis. JAMA 2004;292(13):1573-1580

9 Roetker NS, MacLehose RF, Hoogeveen RC, et al. Prospective study of endogenous hormones and incidence of venous thromboem- bolism: the Atherosclerosis Risk in Communities Study. Thromb Haemost 2018;118(11). Doi: 10.1055/s-0038-1673613

10 Schairer C, Lubin J, Troisi R, Sturgeon S, Brinton L, Hoover R. Menopausal estrogen and estrogen-progestin replacement therapy and breast cancer risk. JAMA 2000;283(04):485-491

11 Suchon P, Al Frouh F, Henneuse A, et al. Risk factors for venous thromboembolism in women under combined oral contraceptive. The PILl Genetic RIsk Monitoring (PILGRIM) Study. Thromb Haemost 2016;115(01):135-142

12 Ay C, Pabinger I, Cohen AT. Cancer-associated venous thromboembolism: burden, mechanisms, and management. Thromb Haemost 2017;117(02):219-230

13 Chung WS, Lin CL. Increased risks of venous thromboembolism in patients with psoriasis. A Nationwide Cohort Study. Thromb Haemost 2017;117(08):1637-1643

14 Hugon-Rodin J, Horellou MH, Conard J, Gompel A, Plu-Bureau G. Type of combined contraceptives, factor $\mathrm{V}$ Leiden mutation and risk of venous thromboembolism. Thromb Haemost 2018;118(05):922-928 